\title{
Literaturhinweise und Abstracts zum Heftthema aus Psycoterapia e Scienze Umane
}

\section{Articoli di Psicoterapia e Scienze Umane sul tema di questo numero}

\section{www.psicoterapiaescienzeumane.it}

\author{
Paolo Migone \\ Psychotherapie-Wissenschaft 8 (2) 63-64 2018 \\ www.psychotherapie-wissenschaft.info \\ CC BY-NC-ND \\ https://doi.org/10.30820/8243.11
}

Carlo Tullio-Altan, Modelli concettuali antropologici per un discorso interdisciplinare tra psichiatria e scienze sociali. Psicoterapia e Scienze Umane, 1967, I, 1: 2-6 [Ristampa in: Psicoterapia e Scienze Umane, 1975, IX, 1: 25-29].

Silvia Montefoschi, Al di là del principio d'autorità. La concezione antropologica di Carl Gustav Jung. Psicoterapia e Scienze Umane, 1969, III, 8/9: 1-24.

Paul Parin, Antropologia e riscostruzione psicoanalitica [edizione originale: Anthropologie und psychoanalytische Rekonstruktion. Schweizerische Zeitschrift für Psychologie und ibre Anwendungen, 1964, 23, 2: 174-180]. Psicoterapia e Scienze Umane, 1971, V, 4: 27-29 (Italian: http:// paul-parin.info/wp-content/uploads/texte/italiano/1971b. pdf; German: http://paul-parin.info/wp-content/uploads/ texte/deutsch/1964a.pdf).

Paul Parin, Critica della società nel processo di interpretazione [Edizione originale: Gesellschaftskritik im Deutungsprozess. Psyche, 1975, 29, 2: 97-117. [Ristampa in: Parin, P. \& Parin-Matthèy, G. (1978). Der Widerspruch im Subjekt. Ethnopsychoanalytische Studien (pp. 34-54). Frankfurt a. M.: Syndikat]. Psicoterapia e Scienze Umane, 1976, X, 1/2: 1-9 [Ristampa (con una nota redazionale) in: Psicoterapia e Scienze Umane, 2009, XLIII, 3: 235-248] (Italian: http://paul-parin.info/ wp-content/uploads/texte/italiano/1976b.pdf; German: http://paul-parin.info/wp-content/uploads/texte/deutsch/1975a.pdf).

Tullio Aymone, Un intervento sull'articolo di P. Parin "Critica della società nel processo di interpretazione». Psicoterapia e Scienze Umane, 1976, X, 1/2: 9-13.

Goldy Parin Matthey \& Paul Parin, Diversità tipiche fra membri della piccola borghesia tedesca meridionale e svizzera: un'indagine etnopsicoanalitica [da: Psyche, 1976, 30, 11: 1028-1047]. Psicoterapia e Scienze Umane, 1977, XI, 3: 1-11 (Italian: http://paul-parin.info/wp-content/uploads/texte/italiano/1977b.pdf; German: http:// paul-parin.info/wp-content/uploads/texte/deutsch/1976c. pdf; English: http://paul-parin.info/wp-content/uploads/ texte/english/1978b.pdf).

Henry Collomb, I modelli della psichiatria africana [Comunicazione al Simposio di Psichiatria Transculturale dell'Avana, 20-26 febbraio 1977]. Psicoterapia e Scienze Umane, 1978, XII, 2: 1-7.

Alberto Panza \& Maurizio Bonicatti, Antropoanalisi del totemismo. Psicoterapia e Scienze Umane, 1982, XVI, 1: 67-79.

Paul Parin, «The mark of oppression»: Studio etnopsicoanalitico su ebrei e su omosessuali in una cultura relativamente permissiva. Psicoterapia e Scienze Umane, 1985, XIX, 4: 3-27 (Italian: http://paul-parin.info/wp-content/ uploads/texte/italiano/1985e.pdf; German: http://paul-parin.info/wp-content/uploads/texte/deutsch/1985b.pdf; English: http://paul-parin.info/wp-content/uploads/texte/ english/1989f.pdf).

Antonella Mancini, Segnalazione di: Julian Leff, Psichiatria e cultura. Una prospettiva transculturale. Torino: Sonda, 1992 (ediz. orig.: Psychiatry Around the Globe: A Transcultural View. London: Gaskell, 1988). Psicoterapia e Scienze Umane, 1993, XXVII, 1: 146.

Salvatore Inglese, Psicoanalisi pagana. Profilo della ricerca e della pratica etnopsichiatrica di Tobie Nathan. Psicoterapia e Scienze Umane, 1994, XXVIII, 4: 69-72.

Antonella Mancini, Segnalazione di: Roberto Beneduce, Frontiere dell'identità e della memoria. Etnopsichiatria e migrazioni in un mondo creolo. Con un saggio di Mahfound Boucebci. Milano: FrancoAngeli, 1998. Psicoterapia e Scienze Umane, 1998, XXXII, 4: 150-151.

Antonella Mancini, Segnalazione di: Alfredo Ancora, La dimensione transculturale della psicopatologia. Uno sguardo da vicino. Prefazione di Julian Leff. Postfazione di Bruno Callieri. Roma: EUR, 1997. Psicoterapia e Scienze Umane, 1998, XXXII, 1: 158. 
Alessandro Taurino, Il significato del corpo anoressico nel discorso sociale contemporaneo. Una riflessione psicoantropologica. Psicoterapia e Scienze Umane, 2003, XXXVII, 3: 79-96.

Antonella Mancini, Segnalazione di: Dela Ranci (a cura di), Questioni di etnopsichiatria clinica. L'esperienza di Terrenuove. Contributi di S. Inglese, S. Ligabue, F. Sironi, D. Ranci, M. Mazzetti, A. Casartelli \& G. Macchieraldo. Milano: Edizioni di Terrenuove, 2004, pp. 160, 14,50 EUR. Psicoterapia e Scienze Umane, 2005, XXXIX, 4: 572.

Paolo Francesco Peloso, Recensione di: Roberto Beneduce, Etnopsichiatria. Sofferenza mentale e alterità fra storia, dominio e cultura. Roma: Carocci, 2007, pp. 399, 24,50 EUR. Psicoterapia e Scienze Umane, 2008, XLII, 3: 420-422.

Pietro Pascarelli, Recensione di: Alberto Merini, Luca Malaffo \& Federica Salvatori, Il bianco e il nero. Esperienze di etnopsichiatria nel servizio pubblico. Bologna: CLUEB, 2008, pp. 232, 16,00 EUR. Psicoterapia e Scienze Umane, 2009, XLIII, 3: 419-422.

Carlotta Gentili, Recensione di: Marie Rose Moro, Quitterie De La Noë, Yoram Mouchenik \& Thierry Baubet, Manuale di psichiatria transculturale. Dalla clinica alla società. Trad. di Giulia Magnani. Milano: FrancoAngeli, 2009, pp. 315, 35,00 EUR (ediz. orig.: Manuel de psychiatrie transculturelle. Travail clinique, travail social. Grenoble: Editions La Pensée Sauvage, 2006; I ediz.: 2004). Psicoterapia e Scienze Umane, 2010, XLIV, 1: 122-125.

Caterina Quarello, Recensione di: Salvatore Inglese \& Giuseppe Cardamone, Déjà Vu. Tracce di etnopsichiatria critica. Paderno Dugnano (MI): Edizioni Colibrì, 2010, pp. 352, 24,00 EUR. Psicoterapia e Scienze Umane, 2011, XLV, 4: 592-593.

Pietro Pascarelli, La psicosomatica nel paradigma biomedico e nella prospettiva dell'antropologia medica [Intervento di discussione della relazione di Piero Porcelli «Sviluppi contemporanei della psicosomatica» tenuto ai «Seminari Internazionali di Psicoterapia e Scienze Umane», Bologna, 19 maggio 2012 (pubblicato a pp. 359-388 di questo n. 3/2012 della rivista)]. Psicoterapia e Scienze Umane, 2012, XLVI, 3: 389-402. https:// doi.org/10.3280/PU2012-003003
Riassunto: Il paradigma biomedico in cui è incardinata la psicosomatica è fondato sulla dicotomia mente-corpo, e su una concezione del corpo astorica e universale. La psicosomatica presenta pertanto una visione asettica e decontestualizzata in cui la malattia e il corpo sono spogliati di ogni valenza simbolica, sociale, politica. La prospettiva dell'antropologia medica apre la strada a un nuovo paradigma, di cui si avverte le necessità, per una lettura critica dei fenomeni in una chiave antidicotomica, in cui il concetto di somatizzazione è sostituito da quello di incorporazione, e il corpo non è più solo superficie di iscrizione dei registri simbolici, ma sede in cui l'economia, il potere e la disuguaglianza mostrano i loro effetti incarnati.

Parole Chiave: psicosomatica, somatizzazione, antropologia medica, incorporazione, paradigma

Psychosomatics within the biomedical paradigm and within the perspective of medical anthropology

The biomedical paradigm, in which psychosomatics is embedded, is based on a mind-body dichotomy and on a universalistic and a historical conception of body. Psychosomatics indeed presents an «aseptic» and decontextualized vision in witch illness and body are deprived of any symbolic, social and political value. The perspective of medical anthropology allows for a new paradigm and a critical reading of clinical phenomenology in a non-dualistic key, where the concept of «somatization» is substituted by the concept of «embodiment». In this view, the body ceases to be a mere inscription of symbolic contents, and becomes the place where economy, power and inequality show their embodied effects.

Key-Words: psychosomatics, somatization, medical anthropology, embodiment, paradigm

Antonella Mancini, Segnalazione di: Stefano Beggiora, Mario Giampà, Alfredo Lombardozzi \& Anthony Molino (a cura di), Sconfinamenti. Escursioni psico-antropologiche. Sesto San Giovanni (MI): Mimesis, 2014, pp. 285, 22,00 EUR. Psicoterapia e Scienze Umane, 2014, XLVIII, 4: 690-691.

Antonella Mancini, Recensione di: Ciro Tarantino \& Alessandra M. Straniero, La bella e la bestia. Il tipo umano nell'antropologia liberale. Sesto San Giovanni (MI): Mimesis, 2014, pp. 143, 14,00 EUR. Psicoterapia e Scienze Umane, 2015, XLIX, 1: 155-158.

Valeria Vaccari, Recensione di: Salvatore Inglese \& Giuseppe Cardamone, Déjà vu 2. Laboratori di etnopsichiatria critica. Presentazione di Gianluca Bocchi \& Mauro Ceruti. Paderno Dugnano (MI): Edizioni Colibrì, 2017, pp. 415, 24,00 EUR. Psicoterapia e Scienze Umane, 2017, 51, 4: 644-646. 